\title{
CARACTERÍSTICAS SEMINAIS E FERTILIDADE EM GARANHÕES
}

\author{
SEMINAL CHARACTERISTICS AND FERTILITY IN STALLIONS
}

\author{
Carlos Eurico Fernandes $^{1} \quad$ Cláudio Alves Pimentel $^{2}$
}

\section{RESUMO}

\begin{abstract}
O objetivo deste experimento foi verificar o efeito da qualidade seminal e sua relação com a fertilidade no garanhão. Os dados foram obtidos de sete haras, incluindo 1198 éguas, 2013 ciclos e 3817 serviços, durante três temporadas consecutivas. A porcentagem de prenhez $(P)$, porcentagem de prenhez por ciclo $(P / C)$ e de serviço $(P / S)$, estimadas para cada garanhão, foram usadas como variáveis dependentes. Adicionalmente, verificou-se o efeito da idade ( $<15$ ou $>15$ anos) e categoria reprodutiva da égua (lactação, falhada ou virgem), sobre os índices reprodutivos. Garanhões com total de espermatozóides viáveis no ejaculado superior a $1,8 \times 10^{9}$ motilidade não inferior a $50 \%$ e percentual de normais acima de $60 \%(n=20)$ apresentaram melhor $P(86 \%$ vs. $74 \%, p<0,01), P / C$ (54\% vs. $45 \%), p<0,01)$ e P/S (28\% vs. $22 \%$, $p<0,01)$ em relação aos garanhões cujas características foram inferiores $(n=16) . O$ percentual de espermatozóides normais correlacionou-se à $P$ (0,33), P/C $(0,50)$ e $P / S(0,36)$, respectivamente. Além de a qualidade seminal, a idade e a categoria reprodutiva das éguas afetaram a fertilidade do garanhão. Esses resultados levam à conclusão de que a qualidade seminal interfere na fertilidade do garanhão; entretanto, outros fatores associados ao manejo reprodutivo, como a idade e a categoria reprodutiva das éguas, são importantes na relação entre qualidade seminal e fertilidade.
\end{abstract}

Palavras-chave : garanhão, sêmen, fertilidade.

\section{SUMMARY}

The objective of this experiment was to verify the effect of sperm quality and its relationship to fertility in stallion. The data was obtained from seven farms and included 36 stallions, 1198 mares, 2013 cycles and 3817 services, during three cosecutive breeding seasons. The overall pregnancy rate $(P)$, pregnancy per cycle $(P / C)$ and pregnancy per service $(P / S)$, for each stallion were used as dependent variables. Additionally, the effect of age of mare ( $<15$ or $>15$ years), mare status (lactating, barren and maiden) on fertility indexes was verified.
Stallions with viable sperm counts higher than $1.8 \times 10^{9}$, motile sperm not lower than $50 \%$ and normals rate higher than $60 \%$ $(n=20)$, exhibited better $P(86 \%$ vs $74 \% p<0.01), P / C(54 \%$ vs $45 \%, p<0.01)$ and $P / S(28 \%$ vs $22 \%)$ than stallions in which sperm characteristics above those standars $(n=16)$. The normals rate was correlated to $P(0.33), P / C(0.50)$ and $P / S(0.36)$, respectively. In addition to sperm quality, age and status mares were affected the fertility of stallions. These results led to the conclusion that seminal quality affects equine fertility; however, other factors associated to reproductive management, age and status mares are important in the relationship between semen quality and fertility.

Key words : stallion, semen, fertility.

\section{INTRODUÇÃO}

A espécie eqüina possui os índices mais baixos de fertilidade quando comparada com as demais espécies domésticas (SULLIVAN et al., 1975; VOSS, 1993). Parte dessas observações relacionam-se ao fato de, que nesta espécie, não há seleção por fertilidade (MERKT, 1986; HUGUES, 1991). Além disso, há uma tendência de se atribuir à fêmea os problemas de infertilidade, comprometendo uma avaliação mais criteriosa envolvendo o macho (PIMENTEL, 1989; HAMES et al., 1996).

Dentre os fatores que podem alterar os índices reprodutivos, a qualidade seminal assume um efeito considerável. No entanto, essa associação tem-se mostrado questionável sob determinados aspectos. BIELANSKI (1951), NISHIKAWA, (1959) e HAAG (1959) foram os pioneiros em

\footnotetext{
${ }^{1}$ Médico Veterinário, MSc, Professor do Departamento de Patologia, CCBS, Universidade Federal do Mato Grosso do Sul, Cidade Universitária, 79070-900, Campo Grande, MS. E-mail: cafernandes@enersulnet.com.br. Autor para correspondência.

${ }^{2}$ Médico Veterinário, PhD., Professor do Departamento de Patologia Animal, Faculdade de Veterinária, Universidade Federal de Pelotas, Pelotas, RS.
} 
estabelecer a variação da fertilidade a partir de diferentes padrões seminais. Enquanto HAAG (1959) verificou que melhores índices de concepção eram obtidos por garanhões que apresentavam mais de $50 \%$ de motilidade e percentuais de espermatozóides anormais inferiores a $40 \%$., BIELANSKI (1975) encontrou garanhões com altos índices de anormalidades morfológicas com excelente fertilidade. Mais tarde, KENNEY et al. (1983) verificaram que a concentração espermática, percentual de espermatozóides morfologicamente normais e a motilidade eram as características seminais que melhor explicavam a variação na taxa de prenhez obtida no fim de uma temporada de monta. Tais características serviram de base para determinar um padrão qualitativo que pudesse auxiliar na interpretação do exame de sêmen. Nesta mesma linha, outros autores têm mostrado que, das variáveis seminais, a motilidade e a morfologia espermática são as mais relevantes (DOWSET $\boldsymbol{e t} \boldsymbol{a l}$. 1984; DAELS et al., 1991; JASKO, 1992). Embora os aspectos morfológicos do sêmen tenham mostrado associações expressivas com a fertilidade (JASKO et al., 1990), as dificuldades em estabelecer um modelo de uso prático na rotina clínica do haras ainda são marcantes. As anormalidades morfológicas variam entre indivíduos, durante o período reprodutivo, e podem ou não estar associadas a outras características seminais (HELLANDER $\boldsymbol{e t} \boldsymbol{a l}$., 1991; VEERAMACHANENI RAO et al., 1993; HAMMES et al., 1996), sem que haja um comprometimento efetivo sobre a fertilidade. Pressupõe-se, com base nesses estudos, que as características seminais assumem um papel decisivo na determinação da fertilidade do garanhão, particularmente na sua eficiência reprodutiva. Porém, muitos aspectos ainda são contraditórios (VOSS et al., 1981; PALMER \& FAUQUENOT, 1985; ROUSSET et al., 1987; AMANN, 1989).

Este trabalho tem como objetivo estudar os efeitos da qualidade seminal de diferentes grupos de garanhões sobre a fertilidade, estimada com base na porcentagem de prenhez, porcentagem de prenhez por ciclo e porcentagem de prenhez por serviço, considerando a idade e a categoria reprodutiva da égua.

\section{MATERIAL E MÉTODOS}

Foram utilizados 19 garanhões, 17 Puro Sangue Inglês (PSI) e dois Crioulos, com idade variando entre seis e 28 anos, com histórico de vida reprodutiva conhecido e sem alterações clínicas detectáveis. Os animais pertenciam a sete propriedades particulares localizadas no Rio Grande do Sul, próximos ao paralelo $32^{\circ}$ e foram estudados durante três temporadas consecutivas (1990 a 1992). As éguas pertenciam a diversas raças, incluindo a PSI, Quarto de Milha, Crioulo e mestiças. Cada égua foi analisada de acordo com seu histórico reprodutivo. As que apresentavam história de problemas reprodutivos específicos ou história de subfertilidade recorrente, não foram consideradas. Os animais foram manejados de acordo com critérios adotados em cada haras, porém todas as éguas foram submetidas ao controle do desenvolvimento folicular por palpação retal com ou sem auxílio da ultra-sonografia. O diagnóstico de gestação foi realizado por palpação retal, aproximadamente aos 20 dias após a ovulação. Nas éguas cobertas durante o cio do potro, o diagnóstico de prenhez foi repetido aproximadamente 10 dias após o primeiro exame, sendo este considerado definitivo. Nas propriedades que possuíam aparelho para ultra-som, o diagnóstico de prenhez foi feito a partir dos 14 dias após a ovulação. Para cada garanhão, calculava-se, no fim do período reprodutivo, a porcentagem de prenhez $(\mathrm{P})$, porcentagem de prenhez por ciclo $(\mathrm{P} / \mathrm{C})$ e porcentagem de prenhez por serviço $(\mathrm{P} / \mathrm{S})$.

O exame de sêmen foi conduzido de acordo com KENNEY et al. (1983) e PIMENTEL (1989). Os garanhões tiveram o sêmen obtido no decorrer do período em que estiveram em serviço, sendo que as colheitas iniciavam a partir do dia 15 de agosto de cada temporada. Após a colheita, anotavam-se os valores do volume (sem gel) e percentual de motilidade. A concentração espermática foi feita em câmara de Neubauer a partir de uma amostra de sêmen sem gel, diluída na proporção 1:20, e preservada em solução de formol salina 1\% (HANCOCK, 1957). O total de espermatozóides ejaculados foi calculado a partir da multiplicação da concentração espermática $\left(\times 10^{6} / \mathrm{m} \ell\right)$ pelo volume de sêmen sem gel. A avaliação da morfologia espermática foi realizada através de esfregaços preparados com sêmen fresco e fixados em chama e por preparações úmidas (contraste de fase), provenientes de amostras mantidas em formol salina tamponada a $1 \%$. Os esfregaços, corados com solução de Eosina Blue a $5 \%$ em álcool metílico, e as preparações úmidas de cada ejaculado eram avaliadas sob imersão, estimando-se os percentuais de espermatozóides morfologicamente normais e com anormalidades de cabeça, peça intermediária, cauda, gota proximal, gota distal, cabeça isolada e acrossomo, em 200 células. O número total de espermatozóides viáveis (TVia) no ejaculado foi determinado de acordo com a seguinte equação (KENNEY et al., 1983): Tvia $=$ Tej. $\times$ Mot. $/ 100 \times N+P I+C I N+C A U / 100$; onde: TVia $=$ Total de Espermatozóides Viáveis (x 109); 
Tej.= Total de Espermatozóides Ejaculados (x 109); Mot. $=$ Porcentagem de células móveis; $\mathrm{N}=$ Porcentagem de Espermatozóides Normais; PI = Porcentagem de Anormalidades de Peça Intermediária; $\mathrm{CIN}=$ Porcentagem de Cabeça Isolada Normal; $\mathrm{CAU}=$ Porcentagem de Anormalidades de Cauda.

Os garanhões foram considerados como observações individuais (casos) a cada temporada reprodutiva (15 de agosto a 15 de dezembro). Assim, no final do período experimental, os dados obtidos totalizaram 36 casos, variando de dois a sete ejaculados para cada caso. Os animais que apresentaram no mínimo 1.8 x $10^{9}$ de espermatozóides viáveis no ejaculado com mais de $50 \%$ de motilidade e $60 \%$ de células morfologicamente normais, constituíram o grupo Q1 (Qualidade 1). Já os animais que apresentaram características seminais com valores inferiores a estes formaram o grupo Q2 (Qualidade 2). As éguas foram classificadas de acordo com a idade $\mathrm{e}$ categoria reprodutiva baseando-se nos critérios de BADI et al. (1981) e WOODS et al. (1987): com menos de 15 anos $(<15)$ e com 15 anos ou mais $(\geq$ $15)$; lactantes (apresentavam potro ao pé), falhadas (vazias da temporada anterior) ou virgens (estavam ingressando na reprodução). Os efeitos da qualidade seminal, idade e categoria reprodutiva da égua, sobre os índices de fertilidade estimados para os garanhões, foram avaliados mediante o teste do ChiQuadrado $\left(\mathrm{x}^{2}\right)$. Através da análise de variância, verificou-se a diferença entre características seminais (transformadas para arco-seno) de acordo com a qualidade seminal de cada grupo (Q1 x Q2). A correlação parcial foi utilizada para determinar a associação entre as médias das características seminais e os índices médios de fertilidade (P, P/C, $\mathrm{P} / \mathrm{S}$ ), tendo cada caso como repetição.

\section{RESULTADOS E DISCUSSÃO}

Os índices reprodutivos verificados neste estudo têm sido propostos para avaliar a eficiência de garanhões ao término de uma estação de monta. Porém, expressam a interação de vários fatores inerentes à fertilidade intrínseca tanto do macho quanto das fêmeas servidas e, ainda, do manejo reprodutivo adotado (PIMENTEL, 1989). Dessa forma, optou-se por avaliar animais submetidos a técnicas comuns, como rufiação, controle do desenvolvimento folicular, diagnóstico precoce de gestação, entre outras. Além disso, as análises foram efetuadas classificando as éguas com base na idade $\mathrm{e}$ categoria reprodutiva, retirando aquelas com problemas reprodutivos que poderiam comprometer o índice final estimado para cada garanhão. Enquanto $\mathrm{P}$ deu uma idéia global da eficiência, P/C possibilitou uma avaliação mais direta de fatores que incidem sobre os resultados, pois considera cada ciclo como unidade de observação para a estimativa da fertilidade, e P/S permitiu o número de serviços (saltos) a que o garanhão foi submetido, estando, portanto, intimamente associada aos critérios adotados em cada haras. Da mesma forma, como em outros estudos, a grande variabilidade na disposição do material experimental torna impossível isolar uma simples variável seminal como a mais importante, sendo muito difícil que um grupo de garanhões apresentem a mesma anormalidade seminal, enquanto outros mantenham níveis dentro de valores normais. Por essas razões, estipulou-se um padrão mínimo para algumas variáveis seminais, abaixo das quais os garanhões supostamente poderiam comprometer a fertilidade (Q2). Os padrões usados (Q1 e Q2) foram baseados em diversos autores (MacLEOD \& McGEE, 1950; KENNEY et al., 1983; DOWSETT \& PATTIE, 1982; PIMENTEL, 1989; JASKO et al., 1990) e foram de utilidade prática para a estimativa da fertilidade potencial do garanhão. $\mathrm{O}$ decréscimo nos índices de fertilidade (Tabela 1) é expressivo, considerando que todos os animais foram submetidos a ótimas condições de manejo, servindo as fêmeas o mais próximo da ovulação (controle folicular). Além disso, em muitas situações, a ultrasonografia foi utilizada para definir o momento da cobertura, permitindo melhores condições à fertilização. Contudo, o efeito da qualidade seminal ficou evidente, já que o grupo Q2 resultou em maior número de ciclos com maior freqüência de coberturas, aspectos contrários à eficiência reprodutiva.

Com base na associação entre variáveis seminais e fertilidade, verificou-se que apenas as características morfológicas estiveram relacionadas à $\mathrm{P}, \mathrm{P} / \mathrm{C}$ e $\mathrm{P} / \mathrm{S}$ (Tabela 1). Os coeficientes obtidos entre normais e $\mathrm{P}(\mathrm{r}=0,33)$ e $\mathrm{P} / \mathrm{S}(\mathrm{r}=0,36)$ indicam que pouco mais de $10 \%$ da variação da fertilidade podem ser explicadas pela simples variação do percentual de normais no ejaculado, valores inferiores aos reportados por PIMENTEL (1989), JASKO et al. (1990) e GASTAL et al. (1991). Porém, ao basearmos a fertilidade na $\mathrm{P} / \mathrm{C}(\mathrm{r}=0,50)$, a estimativa de sua variação em função do percentual de normais se eleva para aproximadamente $25 \%$, sugerindo que este índice seja mais sensível para detectar as variações da fertilidade entre garanhões. Essas observações divergem dos estudos de VOSS et 
Tabela 1 - Valores médios das características seminais de acordo com a qualidade seminal, fertilidade e correlação parcial entre características seminais e índices de fertilidade.

\begin{tabular}{|c|c|c|c|c|c|}
\hline \multirow[b]{2}{*}{ Característica seminal } & \multicolumn{2}{|c|}{ Qualidade seminal } & \multicolumn{3}{|c|}{ Correlação (r) } \\
\hline & Q1 & Q2 & $\mathrm{P}$ & $\mathrm{P} / \mathrm{C}$ & $\mathrm{P} / \mathrm{S}$ \\
\hline Total viáveis(x 1099) & $5,02 \pm 0,6^{\mathrm{A}}$ & $0,78 \pm 0,2^{\mathrm{B}}$ & $\mathrm{ns}$ & $\mathrm{ns}$ & $\mathrm{ns}$ \\
\hline Motilidade $(\%)$ & $61,2 \pm 3,6^{\mathrm{A}}$ & $32,4 \pm 3,5^{\text {в }}$ & $\mathrm{ns}$ & $\mathrm{ns}$ & $\mathrm{ns}$ \\
\hline \multicolumn{6}{|l|}{ Morfologia Espermática (\%) } \\
\hline Normais & $70,2 \pm 2,0^{\mathrm{A}}$ & $55,5 \pm 2,8^{\text {B }}$ & $0,33 *$ & $0,50 *$ & $0,36 *$ \\
\hline Anorm. de Cabeça & $6,4 \pm 0,8 \mathrm{~A}$ & $10,0 \pm 1.1^{\mathrm{B}}$ & ns & ns & $\mathrm{ns}$ \\
\hline Anorm. de Acrossomo & $1,1 \pm 0.2^{\mathrm{C}}$ & $5,8 \pm 2.4^{\mathrm{D}}$ & $-0,50 * *$ & $-0,36^{*}$ & $\mathrm{~ns}$ \\
\hline Anorm. de Peça Intermediária & $2,2 \pm 0.3^{\mathrm{C}}$ & $4,6 \pm 1.0^{\mathrm{D}}$ & $\mathrm{ns}$ & $-0,52 * *$ & $\mathrm{~ns}$ \\
\hline Gota Citoplasmática Proximal & $1,6 \pm 0.3^{\mathrm{C}}$ & $4,4 \pm 1.2^{\mathrm{D}}$ & $\mathrm{ns}$ & ns & $\mathrm{ns}$ \\
\hline Gota Citoplasmática Distal & $3,6 \pm 0.8$ & $3,7 \pm 0.8$ & $\mathrm{~ns}$ & $-0,36^{*}$ & $-0,36 *$ \\
\hline Anorm. de Cauda & $8,7 \pm 1.5$ & $8,2 \pm 1.3$ & $-0,33^{*}$ & $\mathrm{~ns}$ & $\mathrm{~ns}$ \\
\hline Cabeça Isolada Normal & $4,5 \pm 1.2$ & $7,3 \pm 2.7$ & $\mathrm{~ns}$ & $-0,33^{*}$ & $-0,34 *$ \\
\hline \multicolumn{6}{|l|}{ Fertilidade (\%) } \\
\hline $\mathrm{P}$ & $599 / 696(86)^{\mathrm{A}}$ & $389 / 524(74)^{\text {в }}$ & & & \\
\hline $\mathrm{P} / \mathrm{C}$ & $599 / 1108(54)^{\mathrm{A}}$ & $389 / 868(45)^{\text {В }}$ & & & \\
\hline $\mathrm{P} / \mathrm{S}$ & $599 / 2136(28)^{\mathrm{A}}$ & $389 / 1729(22)^{\mathrm{B}}$ & & & \\
\hline
\end{tabular}

\pm erro padrão da média; letras distintas entre colunas representam diferença significativa $(\mathrm{AB}, \mathrm{p}<0,01 ; \mathrm{CD}, \mathrm{p}<0,05)$; $\mathrm{P}=$ porcentagem de prenhez; $\mathrm{P} / \mathrm{C}=$ =porcentagem de prenhez por ciclo; $\mathrm{P} / \mathrm{S}=$ porcentagem de prenhez por serviço.; $* \mathrm{p}<0,01 ; * * \mathrm{p}<0,05$; ns = não significativo.

al. (1981) e PICKETT et al. (1989), que concluíram ser a motilidade a melhor característica seminal para estimar a fertilidade potencial do garanhão. No estudo conduzido por VOSS et al. (1981), foram avaliados apenas três garanhões, sendo que parte do manejo reprodutivo envolvia a inseminação artificial com sêmen congelado. Nas avaliações feitas por PIMENTEL (1989) e GASTAL et al. (1991), embora tivessem encontrado altos coeficientes de correlação, utilizaram um número reduzido de garanhões submetidos a condições homogêneas de manejo. Utilizando um número superior $(n=64)$ e avaliando apenas um ejaculado, JASKO et al. (1990), encontraram uma correlação de $\mathrm{r}=0.36$ $(\mathrm{p}<0,01)$ entre $\mathrm{o}$ percentual de normais e o de prenhez por ciclo, índice inferior ao encontrado neste estudo ( $\mathrm{r}=0,50$, Tabela 1$)$.

Mesmo que a diversidade de manejo a que os animais estejam submetidos seja considerado um aspecto importante no estudo da relação entre fertilidade e qualidade seminal na espécie eqüina (PALMER \& FAUQUENOT, 1985; ROUSSET $\boldsymbol{e t}$ al. 1987), encontrou-se a mesma tendência anteriormente reportada, ou seja, índices mais elevados de concepção e melhor eficiência reprodutiva podem ser esperados com maiores percentuais de espermatozóides normais no ejaculado. Embora a motilidade e o total de espermatozóides viáveis sejam importantes características na determinação da qualidade seminal (KENNEY et al. 1983), os coeficientes de correlação parcial com os índices de fertilidade não foram significativos, revelando que, nas condições deste experimento, os aspectos morfológicos do sêmen foram mais importantes para determinar a variação da fertilidade individual de cada garanhão. A avaliação da motilidade assim como sua inclusão no cálculo do total de espermatozóides viáveis, resulta em estimativas subjetivas, sujeitas à baixa precisão (AMANN, 1989), enquanto as características morfológicas tendem a apresentar padrões mais constantes, com menor variância (PATTIE \& DOWSETT, 1982; JASKO et al., 1991).

Além do percentual de espermatozóides normais, o percentual de anormalidades de acrossomo e peça intermediária foram os mais importantes na correlação com os índices reprodutivos (P/C e P/S). Essas anormalidades freqüentemente são encontradas no sêmen de garanhões com fertilidade reduzida (HURTGEN, 1992), porém estudos reportam casos individuais de anormalidades morfológicas específicas (JOHNSON \& HURTGEN, 1985; HELLANDER $\boldsymbol{e t} \boldsymbol{a l} .$, 1991; FERNANDES \& PIMENTEL, 1997). Os efeitos na fertilidade decorrente da elevação dos percentuais de cabeça isolada normal e gota distal não têm sido descritos. Tais anormalidades, em conjunto com as demais encontradas, podem refletir distúrbios na espermiogênese ou no armazenamento extragonadal, contribuindo para limitar a obtenção de melhores resultados. Contudo, as associações aqui encontradas revelam a necessidade de determinar o percentual das diferentes anormalidades morfológicas encontradas no ejaculado, buscando facilitar a interpretação do espermograma e estimar, com maior chance de acerto, a fertilidade potencial do garanhão. 
Além dos aspectos qualitativos do sêmen dos garanhões, os efeitos da idade e da categoria reprodutiva das éguas foram significativos (Tabela 2) e tornam-se relevantes entre os grupos de garanhões estudados, mostrando que estes fatores estão interagidos com a qualidade seminal. Houve um nítido efeito da qualidade seminal sobre as éguas com menos de 15 anos, quando a fertilidade foi estimada através de $\mathrm{P}$ e $\mathrm{P} / \mathrm{C}$ (Tabela 2). Porém, todos os índices foram inferiores $(p<0,05)$ quando éguas com mais de 15 anos foram servidas por garanhões com baixa qualidade seminal. Embora as condições clínicas do sistema reprodutivo, assim como a fertilidade, começam a decrescer progressivamente a partir dos 15 anos (HUTTON \& MEACHAM, 1968; BADI et al., 1981; CARNEVALE \& GINTER, 1992), os resultados indicam que a qualidade do ejaculado desempenha um papel fundamental na obtenção de índices reprodutivos mais favoráveis, já que as éguas mais velhas, quando cobertas por garanhões com sêmen de alta qualidade, apresentaram níveis satisfatórios de fertilidade $\quad(\mathrm{P}=88 \%, \quad \mathrm{P} / \mathrm{C}=47 \%$ e $\mathrm{P} / \mathrm{S}=26 \%$, Tabela 2). Uma tendência semelhante foi verificada na análise do efeito da categoria reprodutiva sobre a fertilidade. As diferenças foram mais marcantes nos garanhões com baixa qualidade seminal. Neste grupo, éguas virgens apresentaram melhor P (85\%) e P/C (49\%), em comparação com lactantes $(69 \%$ e $41 \%)$ e falhadas $(73 \%$, e $38 \%)$, respectivamente. Essas observações sugerem que tanto a idade quanto a categoria reprodutiva das éguas servidas estão diretamente envolvidas na diferença da fertilidade entre garanhões. No entanto, os aspectos qualitativos do sêmen devem ser considerados criteriosamente nas decisões de manejo, sobretudo nos animais que mantêm uma baixa qualidade seminal.

Conclui-se, com essas observações, que a análise da fertilidade com base na $\mathrm{P} / \mathrm{C}$ é mais importante do que $\mathrm{P}$ para estimar o potencial reprodutivo do garanhão. Os limites mínimos de 1,8 x $10^{9}$ de espermatozóides viáveis, $50 \%$ de motilidade e $60 \%$ de espermatozóides morfologicamente normais no ejaculado, são críticos para a qualidade do ejaculado. Porém, a morfologia espermática pode ser considerada um fator essencial para a obtenção de melhor eficiência reprodutiva.

\section{AGRADECIMENTOS}

Os autores agradecem a colaboração dos Méd. Veterinários Ulisses Carneiro, Walter Flores e Flávio D. De La Corte, pelo auxílio e disponibilidade de dados de campo, e ao Dr. José Carlos F. de Moraes, pela ajuda nas análises estatísticas.
Tabela 2 - Fertilidade dos garanhões de acordo com a qualidade seminal, a idade e a categoria reprodutiva da égua.

\begin{tabular}{|c|c|c|c|}
\hline \multirow[b]{2}{*}{ Idade } & \multicolumn{3}{|c|}{ Fertilidade } \\
\hline & $\mathrm{P}(\%)$ & $\mathrm{P} / \mathrm{C}(\%)$ & $\mathrm{P} / \mathrm{S}(\%)$ \\
\hline $\begin{array}{l}<15 \\
\text { Q1 }\end{array}$ & $508 / 573(89)^{\mathrm{A}}$ & $508 / 906(56)^{\mathrm{A}}$ & $508 / 1777(35)^{\mathrm{A}}$ \\
\hline$>15$ & $96 / 109(88)^{\mathrm{A}}$ & $96 / 206(47)^{\text {в }}$ & $96 / 403(26)^{\mathrm{B}}$ \\
\hline $\begin{array}{l}<15 \\
\mathrm{Q} 2\end{array}$ & $289 / 403(72)^{\text {в }}$ & $289 / 687(42)^{\text {B }}$ & $289 / 1309(34)^{\mathrm{A}}$ \\
\hline$>15$ & $79 / 113(70)^{\text {в }}$ & $79 / 214(37)^{\mathrm{C}}$ & $79 / 404(19)^{\mathrm{C}}$ \\
\hline $\begin{array}{l}\text { Categoria } \\
\text { Lactantes }\end{array}$ & $365 / 426(86)^{A}$ & $365 / 686(53)^{A}$ & $365 / 1282(35)^{\mathrm{A}}$ \\
\hline $\begin{array}{ll}\text { Q1 } & \text { Falhadas } \\
\text { Virgens }\end{array}$ & $\begin{array}{l}172 / 188(91)^{A} \\
66 / 72(92)^{A}\end{array}$ & $\begin{array}{l}172 / 305(56)^{\mathrm{A}} \\
66 / 128(52)^{\mathrm{A}}\end{array}$ & $\begin{array}{l}172 / 594(29)^{\mathrm{A}} \\
66 / 301(22)^{\mathrm{B}}\end{array}$ \\
\hline Lactantes & $234 / 340(69)^{\mathrm{B}}$ & $234 / 572(41)^{\text {B }}$ & $234 / 1060(22)^{\mathrm{B}}$ \\
\hline $\begin{array}{ll}\text { Q2 } & \begin{array}{l}\text { Falhadas } \\
\text { Virgens }\end{array}\end{array}$ & $\begin{array}{l}83 / 114(73){ }^{\mathrm{B}} \\
52 / 61(85)^{\mathrm{A}}\end{array}$ & $\begin{array}{l}83 / 216(38) \text { в } \\
52 / 106(49)\end{array}$ & $\begin{array}{l}83 / 400(21)^{\text {В }} \\
52 / 261(20)^{\text {в }}\end{array}$ \\
\hline
\end{tabular}

Letras diferentes entre linhas representam diferença significativa $(\mathrm{p}<0,05)$, individualmente para idade e categoria reprodutiva.

Q1 e Q2 = qualidade seminal;

$\mathrm{P}=$ porcentagem de produção; $\mathrm{P} / \mathrm{C}=$ porcentagem de produção no ciclo; $\mathrm{P} / \mathrm{S}=$ produção por serviço.

\section{REFERÊNCIAS BIBLIOGRÁFICAS}

AMANN, R.P. Can the fertility potential of seminal sample be predicted accurately? J Androl, v.10, n.2, p.89-98, 1989.

BADI, A.M., O'BYRNE, T.M., CUNNINGHAN, E.P. An analysis of reproductive performance in thoroughbred mares. Irish Vet J, v.1, p.4-12, 1981.

BIELANSKI, W. Characteristics of the semen of stallions. Macro and microscopic investigations with estimation of fertility. Mem Acad Pol Sci, v.16, p.1-58, 1951.

BIELANSKI, W. The evaluation of stallion semen in aspects of fertility control and its use for artificial insemination. $\mathbf{J}$ Reprod Fertil, Suppl.23, p.19-23, 1975.

CARNEVALE, E.M., GHINTER, O.J. Relationships of age to uterine function and reproductive efficiency in mares. Theriogenology, v.36, p.1101-1115, 1992.

DAELS, P.F., HUGHES, J.P., STABENFELDT, G.H. Reproduction in horses. In: CUPPS, P. T. Reproduction in domestic animals. 4.ed. San Diego : Academic, 1991. p.413-444.

DOWSETT, K.F., PATTIE, W.A. Characteristics and fertility of stallion semen. J Reprod Fertil, Suppl.32, p.1-8, 1982.

DOWSETT, K.F., OSBORNE, H.G. PATTIE, W.A. Morphological characteristics of stallion's spermatozoa. Theriogenology, v.22, n.5, p.463-472, 1984.

FERNANDES, C.E., PIMENTEL, C.A. Anormalidades de acrossomo e fertilidade em um garanhão: relato de um caso. Ciência Rural, v.27, n.2., p.345-349, 1997. 
GASTAL, E.L., AUGUSTO, C., CASTRO, T.A. M. G. et al. Relação entre qualidade espermática e fertilidade em garanhões. In: CONGRESSO BRASILEIRO DE REPRODUÇÃO ANIMAL, 9, 1991, Belo Horizonte, MG. Anais... Belo Horizonte :CBRA, 1991. V.II, p.446.

HAAG, F.M. Evaluation of "dismount" semen in thoroughbred horse breeding. J Amer Vet Med Assoc, v.1:312-314, 1959.

HAMMES, A.M., PIMENTEL, C.A., FERNANDES, C.E. Fertilidade em garanhões avaliada através do exame andrológico. Ciência Rural, v.26, n.2., p.277-283, 1996.

HANCOCK, J.L. The morphology of boar spermatozoa. J Microsc Soc, v.76, p.84-97, 1957.

HELLANDER, J.C., SAMPER, J.C., CRABO, B.G. Fertility of a stallion with low sperm motility and a height incidence of an unusual sperm tail defect. Vet Rec, v.128, p.449-451, 1991.

HUGHES, J.P. Curso de Eqüinos. In: CONGRESSO BRASILEIRO DE REPRODUÇÃO ANIMAL, 9, 1991, Belo Horizonte. Anais... Belo Horizonte : CBRA, 1991.

HURTGEN, J.P. Evaluation of the stallion for breeding soundness. The Veterinary Clinics of North America: Equine Practice, v.8, n.1, p.149-165, 1992.

HUTTON, C.A., MEACHAM, T.N. Reproductive efficiency on fourteen horse farms. J Anim Sci, v.27, p.434-438, 1968.

JASKO, D.J. Evaluation of stallion semen. The Veterinary Clinics of North America: Equine Practice, v.8, n.1, p.129-148, 1992.

JASKO, D.J., LEIN, D.H., FOOT, R.H. Determination of the relationship between sperm morphologic classifications and fertility en stallions: 66 cases (1987-1988). J Amer Vet Med Assoc, v.197, n.3, p.389-394, 1990

JASKO, D.J.; LEIN, D.H., FOOTE, R.H. The repeatability and effect of season on seminal characteristics and computer-aided sperm analysis in the stallion. Theriogenology, v.35, n.2, p.317-327, 1991.

JOHNSON, L.A., HURTGEN, J.P. The morphological and ultrastrutural appearance of the crater defect in stallion spermatozoa. Gamete Research, v.12, p.41-46, 1985.

KENNEY, R.M., HURTGEN, J.P.; PERSON, R., et al. Society for theriogenology: manual for clinical fertility evaluation of the stallion. Hastings, NB : ni, 1983 . $100 \mathrm{p}$

MacLEOD, J., McGEE, W.R. The semen of the thoroughbred. Cornell Vet, v.40, p.233-248, 1950.
MERKT, H. Exame andrológico e problemas de cobertura no garanhão. Esquema para o exame andrológico. In ENCONTRO NACIONAL DE EQÜIDEOCULTURA, 4, 1986, São Paulo, SP. Anais... São Paulo : Sociedade Brasileira de Hipologia, 1986. p.33-34.

NISHISAWA, Y. Studies on reproduction in horses. Singularity and artificial control in reproductive phenomena. Tokyio, Japan : Koei Kvoto, 1959. 340p.

PALMER, W.A., FAUQUENOT, A. Mesure et prédiction de la fertilite des étalons. Etude méthodologie. Nouzilly, France: INRA, 1985. p.113-127.

PATTIE, W.A., DOWSETT, K.F. The repeatability of seminal characteristics of stallions. J Reprod Fertil, Supl.32, p.1-13. 1982.

PICKETT, B.W. AMANN, R.P. McKINNON, A.O. et al. Manegement of the stallion for maximum reproductive efficiency, II. Fort Collins : Anim Reprod Laborat, CO., 1989. 126p.

PIMENTEL, C.A. Aspectos da patologia espermática e a fertilidade no garanhão. In: CONGRESSO BRASILEIRO DE REPRODUÇÃO ANIMAL, 1989, Belo Horizonte, MG. Anais... Belo Horizonte : CBRA, 1989. V.8, p.127-132.

ROUSSET, H., CHANTELAUBE, C.; MAGISTRINE, M. et al. Assessment of fertility and semen evaluation of stallion. $\mathbf{J}$ Reprod Fertil, Supl.35, p.25-31, 1987.

SULLIVAN, J.J., TURNER, p.C.; SELF, L.C., et al. Survey of reproductive efficiency in the quarter horse and thoroughbred. J Reprod Fertil, Supl.23, p.315-318, 1975.

VEERAMACHANENI RAO, D.N., MOELLER, C.L., PICKETT, B.W. et al. On processing and evaluation of equine seminal samples for cytopathology and fertility assessment: the utility of electron microscopy. J Equi Vet Sci, v.13, n.4. 207-215, 1993.

VOSS, J.L. Breeding Efficiency. In: McKINNOW, A.O., VOSS, J.L. Equine reproduction. Phyladelphya : Lea \& Febiger. 1993. 1114p.

VOSS, J.L., PICKETT, B.W., SQUIRES, E.L. Stallion spermatozoa morphology and motility and their relationship to fertility. J Amer Vet Med Assoc, v.199, n.12, p.1760-1761, 1981.

WOODS, G.L., BAKER, C.B., BALDWINT, J.L., et al. Early pregnancy loss in brood mares. J Reprod Fertil, Supl.35, p.455-459, 1987. 